\title{
Periodic and Nonperiodic Phenomena in AGB Stars ${ }^{1}$
}

\author{
T. Lebzelter \\ Institut f. Astronomie, University of Vienna, Austria
}

K.H. Hinkle

National Optical Astronomy Observatories ${ }^{2}$, Tucson, USA

\begin{abstract}
We discuss velocity variations in different layers of an AGB star's atmosphere. Periodic and nonperiodic changes are observed. From a large sample of data we derive mean velocity curves for miras and semiregular variables.
\end{abstract}

\section{Introduction}

The structure of the atmospheres of Asymptotic Giant Branch (AGB) stars is dominated by stellar pulsations and therefore varies strongly during the light cycle. High resolution infrared spectroscopy has turned out to be a very powerful tool to investigate the dynamics of AGB star atmospheres caused by the stellar pulsation (Hinkle et al., 1982). The rich vibrational-rotational molecular spectrum of these stars offers a wide variety of lines of different excitation originating in different layers of the atmosphere. These lines can be used to monitor the dynamics at different depths in the stellar atmosphere. However, so far only a small sample of AGB variables and only a small number of molecular species have been investigated in detail (e.g. Hinkle et al., 1984).

In this paper we present a summary of our recent work on the dynamics of AGB star atmospheres obtained by means of time series high resolution infrared spectra of a large sample of AGB stars. We extended the existing data material significantly, investigating the behavior of a large variety of molecular lines in AGB variables of different types.

\section{Observations}

Time series of high resolution spectra of several parts of the 1.5 to $4 \mu \mathrm{m}$ region have been obtained at Kitt Peak National Observatory with the FTS, the NICMASS- and the PHOENIX-spectrograph. Spectral resolution was typically 40000 or better. Line positions have been measured for a selection of atomic

\footnotetext{
${ }^{1}$ Based on observations obtained at Kitt Peak National Observatory

${ }^{2}$ operated by the Association of Universities for Research in Astronomy, Inc. under cooperative agreement with the National Science Foundation
} 

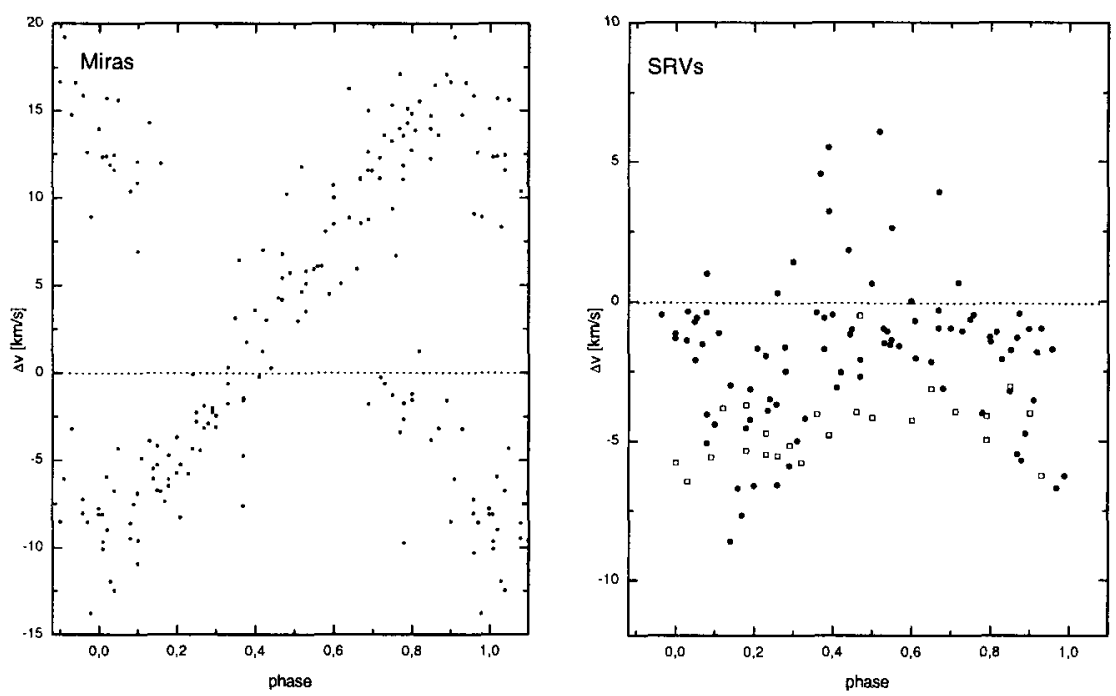

Figure 1. Left: Velocity variations in Mira-type variables relative to the center of mass velocity versus phase. Phases are derived from AAVSO lightcurves. Right: Velocity variations in semiregular variables relative to the center of mass velocity versus phase. Phases are estimated from nearby light maxima or minima observed with an automatic telescope. Open symbols denote the time series of observations of W Cyg indicating an extreme case for the asymmetry relative to the center of mass velocity.

and molecular species. The derived velocities have an accuracy of at least 0.3 $\mathrm{kms}^{-1}$. Several lines exhibit at some phases multiple absorption and/or emission components. Each of these components has been measured. Their reality has been checked using averaged line profiles (Lebzelter, Hinkle, \& Aringer 2001).

\section{Results}

The deep atmospheric layers of miras monitored by e.g. high excitation CO lines at $1.6 \mu \mathrm{m}$ show a periodic variation with typical indications of radial pulsation and the occurence of shocks (discontinuous velocity curves). They indicate the pulsation of the star that is also visible in the visual light change. With the help of our large sample we are now able to derive mean velocity curves for these layers in LPVs. All miras (Fig.1, left panel) show the same type of velocity variations. Velocity amplitudes are very similar. Our sample of miras covers a period range between 145 and 470 days, and includes stars of spectral type $\mathrm{M}$, $\mathrm{S}$ and $\mathrm{C}$. No dependency on period, metallicity or chemistry was found (Hinkle et al., 1997; Lebzelter et al., 1999). 


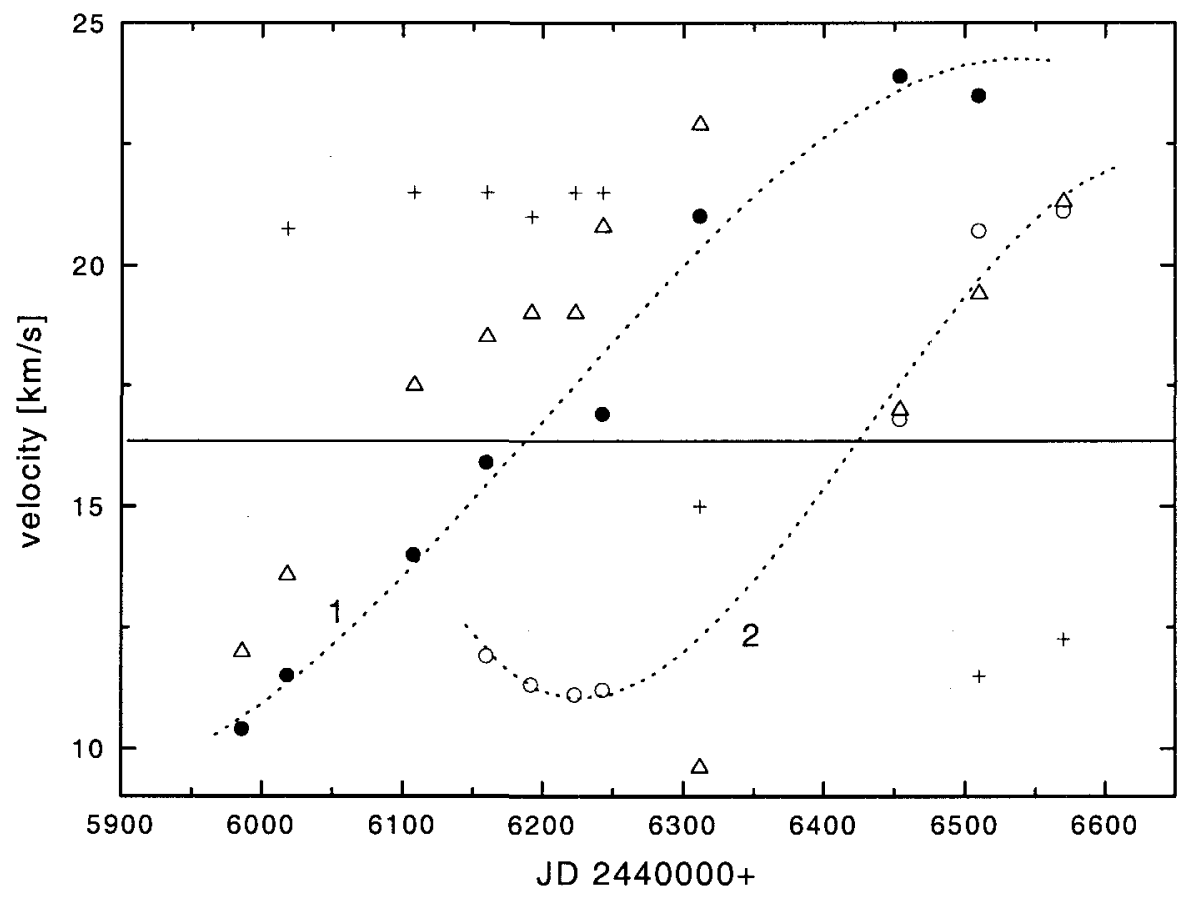

Figure 2. $\quad \mathrm{H}_{2} \mathrm{O}$ and $\mathrm{OH}$ velocities of $\mathrm{R}$ Cas versus time. Filled and open circles mark two different $\mathrm{H}_{2} \mathrm{O}$ absorption components, crosses indicate water emission lines, open triangles denote $\mathrm{OH}$ absorption. Two velocity curves have been drawn through the $\mathrm{H}_{2} \mathrm{O}$ data points. The horizontal line marks the centers of mass velocity.

Semiregular variables (SRVs) have continuous velocity curves with the maximum velocity occuring at light minimum (Fig. 1, right panel). The velocity changes show the same type of semiregular behaviour found in the light curves of these stars. Amplitudes range from 2 to $15 \mathrm{kms}^{-1}$ (Hinkle et al. 1997; Lebzelter 1999). A strong asymmetry relative to the center of mass velocity is observed in most of the SRVs which is not understood yet (Lebzelter, 1999, Lebzelter et al., 2000). One possible explanation, long term variations due to pulsation or binarity, is discussed by Lebzelter et al. (these proceedings).

The outer atmospheric layers of these stars, investigated with the help of molecules like $\mathrm{SiO}$ or $\mathrm{H}_{2} \mathrm{O}$, do not always follow the period defined by the visual light change, i.e. line profiles obtained at similar phases can differ significantly from each other. These variations may be nonperiodic or periodic on a longer timescale than the main period. An example is given in Fig. 2 for the velocities of high excitation $\mathrm{H}_{2} \mathrm{O}$ lines in $\mathrm{R}$ Cas plotted against time. It can be seen that the velocity curve starting at the beginning of the observed time series shows the same shape as the typical mira velocity curves, but lasts considerably longer than 
the light cycle. The next cycle shows again a velocity curve following the stellar period. The observed difference between the two cycles indicates that either the accelaration of the $\mathrm{H}_{2} \mathrm{O}$ line forming layer was higher or the deceleration lower. Both possibilities may be connected to a non-continuous mass loss in this star (Lebzelter et al., 2001). A similar behaviour was found in a second star, $\chi$ Cyg. In both cases the outstanding velocity behaviour followed a very bright light maximum. This suggests that these two phenomena are related to each other. Richter \& Wood (2001) showed a similar connection between bright maxima and outstanding behaviour of the UV spectrum which is, as the $4 \mu \mathrm{m}$ lines, formed in the outer parts of the atmosphere. However, this phenomenon seems to affect only the outer layers of the star as such a behaviour is not observed e.g. in the $1.6 \mu \mathrm{m}$ CO lines. It is therefore not due to a stronger pulsation of the star.

\section{Conclusions and outlook}

Periodic and nonperiodic velocity variations are found in AGB variables. The inner layers close to the pulsation driving zone show a periodic and highly similar velocity curve in all types of miras. This fact makes it very unlikely that some of the miras pulsate in a different pulsation mode than the others as has been discussed in the literature (e.g. Barthes, 1998). In the outer layers nonperiodic effects occur and the observed stars show a strong diversity in their line profile changes.

Our observational results allow a first overview on the pulsational properties of the whole atmosphere of an AGB star. This can now be compared with recent dynamic model atmospheres (Höfner, 1999). Both periodic and nonperiodic phenomena are predicted by the models. A detailled comparison of models and observations has been started.

\section{References}

Barthes, D. 1998, A\&A, 333, 647

Hinkle, K.H., Hall, D.N.B., \& Ridgway, S.T. 1982, ApJ, 252, 697

Hinkle, K.H., Scharlach, W.W.G., \& Hall, D.N.B. 1984, ApJS, 56, 1

Hinkle, K.H., Lebzelter, T., \& Scharlach, W.W.G. 1997, AJ, 114, 2686

Höfner, S. 1999, A\&A, 346, L9

Lebzelter, T., 1999, A\&A, 351, 644

Lebzelter, T., Hinkle, K.H., \& Hron, J. 1999, A\&A, 341, 224

Lebzelter, T., Kiss, L.L., \& Hinkle, K.H. 2000, A\&A, 361, 167

Lebzelter, T., Hinkle, K.H., \& Aringer, B. 2001, A\&A, in press

Richter, He., \& Wood, P.R. 2001, A\&A, 369, 1027 